$$
\text { "kovacs" — 2006/6/22 — 16:26 — page } 101 \text { — \#1 }
$$

\title{
On the fixed points of an affine transformation: an elementary view
}

\author{
ZOLTÁn KOvÁCS
}

Abstract. This note shows how the fixed points of an affine transformation in the plane can be constructed by an elementary geometric method. The approach presented here also shows how the affinities of the plane can be classified with the help of their fixed points.

Key words and phrases: fixed point theorem, affine transformation, equiaffine transformation.

ZDM Subject Classification: G05, G45, G75.

\section{Introduction}

Every mathematics major college student has to be known the fixed point theorem of the similarities of $\mathbb{R}^{n}$ : if a similarity of $\mathbb{R}^{n}$ is not an isometry, then it has a unique fixed point. Perhaps most of them adds also the argument: the statement is a simple consequence of the famous Banach fixed point theorem for contractions in a complete metric space. However, the fixed points not only of similarities but also the broader class of affine transformations in $\mathbb{R}^{n}$ can be studied with more elementary tools.

This note shows how the fixed points of an affine transformation in the plane can be constructed by an elementary geometric method. I learned the base idea from [1], where an elementary ruler construction is given for the fixed point of a similarity in the plane. However, this construction can be easily applied for 


$$
\text { "kovacs" — 2006/6/22 — 16:26 — page } 102 \text { — \#2 }
$$

affine transformations of the plane, too. Perhaps this fact was well-known in the "golden age" of classical projective geometry, but its earliest source I found in [6].

This approach also shows how the affinities of the plane can be classified with the help of their fixed points.

I have successfully presented this treatment several times, in a geometry course for mathematics majors who have studied linear algebra.

\section{Affine transformations}

Basic properties of affine transformations are well-known (reference [5] is a good analytic introduction), however, for the readers' convenience, I recall some basic notions.

A bijective transformation $F: \mathbb{R}^{n} \rightarrow \mathbb{R}^{n}$ is called affine, if

$$
\forall t \in \mathbb{R}, \forall x, y \in \mathbb{R}^{n}: \quad F(t x+(1-t) y)=t F(x)+(1-t) F(y),
$$

or equivalently $F=\tau_{v} \circ \phi$ for a unique linear automorphism $\phi \in \operatorname{Gl}\left(\mathbb{R}^{n}\right)$, and for a unique translation

$$
\tau_{v}: \mathbb{R}^{n} \rightarrow \mathbb{R}^{n}, \quad x \mapsto x+v, \quad\left(v \in \mathbb{R}^{n}\right) .
$$

$\phi$ is called the linear part of $F$.

A vector $x \in \mathbb{R}^{n}$ is a fixed point of $F$ (i.e., $F(x)=x$ ) if and only if $\phi(x)+v=x$, or in a more illuminating form, if

$$
(\phi-\mathrm{id})(x)=-v \text {. }
$$

(2) can be recognized as a system of linear equations, so in the solvable case rank $r$ of the linear operator $\phi-$ id says everything on the structure of fixed points, namely the set of all fixed points is an affine subspace of dimension $n-r$ in $\mathbb{R}^{n}$. Of course in case of $r=n$ one does not need to assume the solvability of (2), and one gets immediately the fixed point theorem of affine transformations:

Theorem 1. An affine transformation $F=\tau_{v} \circ \phi$ has a unique fixed point if and only if $\phi-i d$ is an isomorphism of $\mathbb{R}^{n}$. Moreover, the unique fixed point is $(\phi-i d)^{-1}(-v)$.

COROLlary 1 (Fixed point theorem of similarities). If a similarity of $\mathbb{R}^{n}$ is not an isometry, then it has a unique fixed point. 
Proof. Let $F$ be a similarity with magnification factor $1 \neq \lambda>0$. The linear part of $F$ is $\lambda \psi$, where $\psi$ is an orthogonal transformation of $\mathbb{R}^{n}$. We need only to show that the kernel of $\lambda \psi-$ id contains the zero vector alone. If $x \in \mathbb{R}^{n}$ is a vector in the kernel of $\lambda \psi-\mathrm{id}$, then $\lambda \psi(x)=x$, therefore $\lambda \cdot\|\psi(x)\|=\|x\|$. Since $\psi$ preserves the norm, we have $(\lambda-1)\|x\|=0$, hence $x=0$.

The fundamental theorem of the plane-affine geometry states that any two triangles (or equivalently, any two parallelograms) of the plane can be related by a unique affine transformation. This theorem enables us to define a plane affine map through the assignment of the related triangles. For example, given a line $t$ and two points $p, q$ off $t$, there is a unique affine transformation that leaves every point on $t$ fixed, and sends $p$ to $q$. This affine transformation is denoted by $[t, p \mapsto q]$, and is called an axial affine transformation.

\section{The construction}

Hereafter for $x \neq y \in \mathbb{R}^{2}$ the line $t x+(1-t) y(t \in \mathbb{R})$ will be denoted by $x y$, while the segment $t x+(1-t) y(t \in[0,1])$ by $[x y]$.

ThEOREM 2. Let $F: \mathbb{R}^{2} \rightarrow \mathbb{R}^{2}, z \mapsto F(z)=z^{\prime}$ be an affine transformation of the plane. Let $(a, b, c, d)$ be a parallelogram i.e., let $a, b, c, d \in \mathbb{R}^{2}$ non-collinear points such that $b-a=c-d$. Suppose, moreover, that

$$
\operatorname{rank}\left(b-a, b^{\prime}-a^{\prime}\right)=\operatorname{rank}\left(d-a, d^{\prime}-a^{\prime}\right)=2 .
$$

Let, finally, $a b \cap a^{\prime} b^{\prime}=p, d c \cap d^{\prime} c^{\prime}=r, b c \cap b^{\prime} c^{\prime}=q, a d \cap a^{\prime} d^{\prime}=s$. With these notations, we have:

(1) if $F$ has a unique fixed point $m$, then $m=p r \cap q s$,

(2) if $F$ has no fixed point, then $p r \| q s$ but $p r \neq q s$,

(3) if $F$ has a pointwise-fixed line, then this line is $p r=q s$,

(Figure 1).

I note that the proof of Theorem 2 in [6] computes the equations of lines $p r$ and $q s$ in a special coordinate-system. From these equations a system of linear equations is formed, and the equivalence of this system and (2) is proven. The following pure analytic proof differs from the one presented in [6], and tries to grasp the geometric idea of the proof in [1]. 

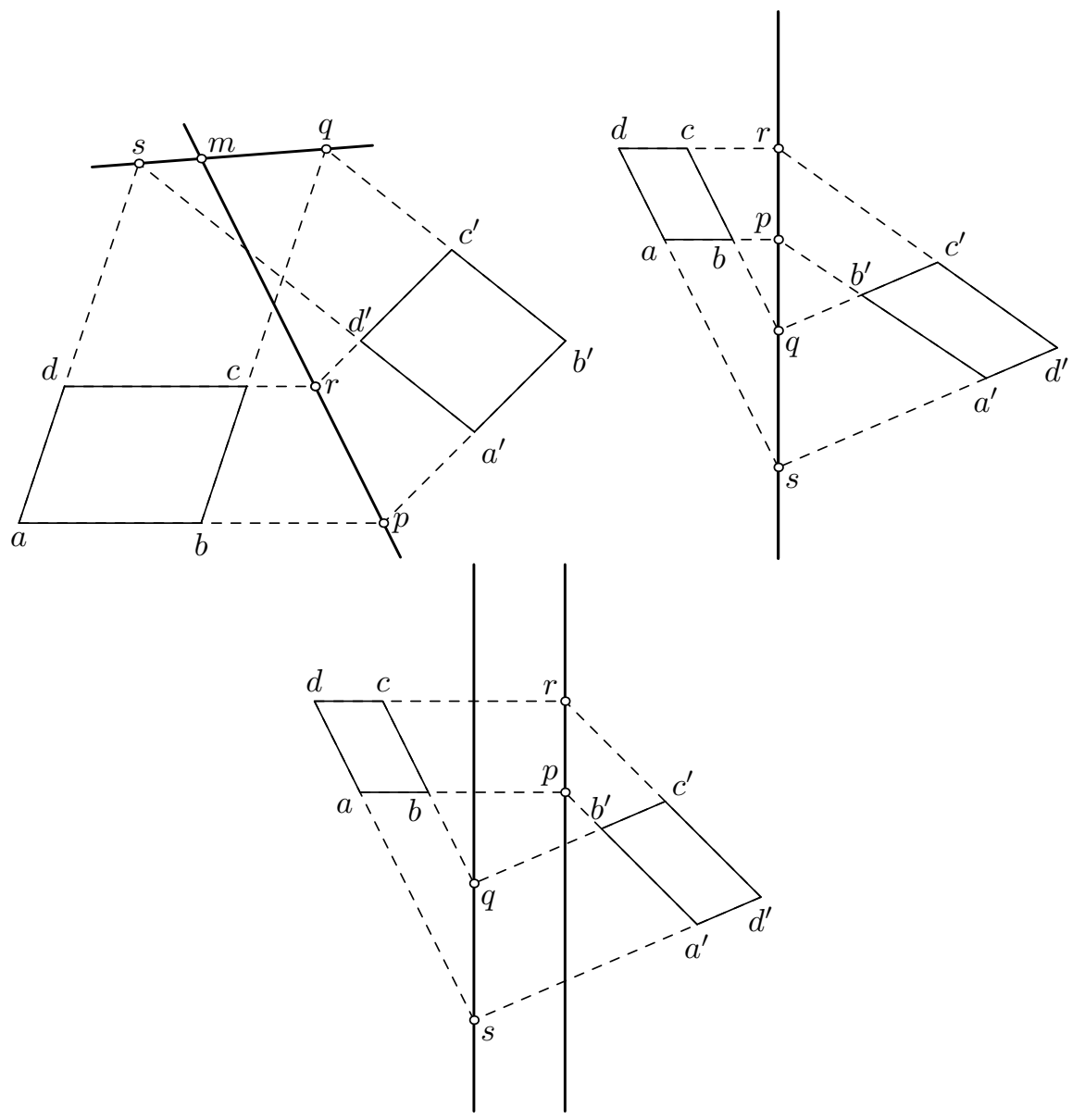

Figure 1. Ruler construction for fixed points of an affine transformation in the plane

Proof. The third statement and its converse is obvious. If $F$ is an axial affine transformation, then the corresponding lines meet each other on the axis. Conversely, if $p r=q s$ then for some $t \in \mathbb{R}$ we have

$$
p=t a+(1-t) b
$$

Since $c b \| a d$ and $c^{\prime} b^{\prime} \| d^{\prime} a^{\prime},(3)$ implies:

$$
p=t s+(1-t) q \Longrightarrow p=t a^{\prime}+(1-t) b^{\prime} \Longrightarrow p=p^{\prime},
$$




$$
\text { "kovacs" — 2006/6/22 — 16:26 — page } 105-\# 5
$$

and analogously $r=r^{\prime}$. Thus the line $p r=q s$ is pointwise fixed.

Now we prove the first statement. From the definition of points $p$ and $r$, it follows that there exist $\alpha, \beta, \gamma, \delta \in \mathbb{R}$, such that:

$$
\begin{aligned}
& p=\alpha a+(1-\alpha) b=\beta a^{\prime}+(1-\beta) b^{\prime} \\
& r=\gamma d+(1-\gamma) c=\delta d^{\prime}+(1-\delta) c^{\prime}
\end{aligned}
$$

that is

$$
\begin{aligned}
& p^{\prime}=\alpha a^{\prime}+(1-\alpha) b^{\prime} \\
& r^{\prime}=\gamma d^{\prime}+(1-\gamma) c^{\prime}
\end{aligned}
$$

From (6) and (4) we obtain that

$$
p^{\prime}-p=(\alpha-\beta)\left(a^{\prime}-b^{\prime}\right)
$$

Similarly, from (7) and (5) we get

$$
r^{\prime}-r=(\gamma-\delta)\left(d^{\prime}-c^{\prime}\right)
$$

Since $\left(a^{\prime}, b^{\prime}, c^{\prime}, d^{\prime}\right)$ is a parallelogram, it follows that

$$
a^{\prime}-b^{\prime}=d^{\prime}-c^{\prime}=x .
$$

For some $t \in \mathbb{R}$, multiply (8) by $t$ and $(9)$ by $(1-t)$. This leads to

$$
\begin{gathered}
t p^{\prime}-t p=t(\alpha-\beta) x \\
(1-t) r^{\prime}-(1-t) r=(1-t)(\gamma-\delta) x .
\end{gathered}
$$

Now, adding (11) and (12) we obtain

$$
t p^{\prime}+(1-t) r^{\prime}=t p+(1-t) r+[t(\alpha-\beta)+(1-t)(\gamma-\delta)] x .
$$

Observe that if $\phi-$ id is a linear automorphism, then we can determine $t$ such that

$$
t(\alpha-\beta)+(1-t)(\gamma-\delta)=0 \Longleftrightarrow t[(\alpha-\beta)-(\gamma-\delta)]=-(\gamma-\delta) .
$$

Indeed, arguing by contradiction, if $(\alpha-\beta)-(\gamma-\delta)=0$ then from (8) and (9) we get

$$
p^{\prime}-p=r^{\prime}-r \Longrightarrow \phi(p)-p=\phi(r)-r \Longrightarrow(\phi-\mathrm{id})(p-r)=0,
$$




$$
\text { "kovacs" — 2006/6/22 — 16:26 — page } 106 \text { — \#6 }
$$

which is impossible. With

$$
t=-\frac{\gamma-\delta}{(\alpha-\beta)-(\gamma-\delta)},
$$

formula (13) reduces to

$$
t p^{\prime}+(1-t) r^{\prime}=t p+(1-t) r .
$$

The point so obtained is denoted by $m$. Now applying $F$ to $m$ we get

$$
F(m)=F(t p-(1-t) r)=t p^{\prime}+(1-t) r^{\prime}=m .
$$

It means that the fixed point $m$ is on the line $p r$, and analogously on $q s$. Thus the first statement is proved.

Further analysis of (13) leads to the second statement. If $\alpha-\beta=\gamma-\delta=0$, then from (8) and (9) we get $p^{\prime}=p$ and $r^{\prime}=r$, meaning that $F$ leaves $p$ and $r$ fixed. If an affine transformation leaves fixed two distinct points, then it leaves fixed every point on the joining line of these points. This is case 3 , so if $F$ has no fixed point, then $\alpha-\beta=\gamma-\delta \neq 0$. From (8) and (9) we get

$$
\begin{gathered}
p^{\prime}-p=r^{\prime}-r \Longleftrightarrow \phi(p-r)=p-r \Longleftrightarrow \\
\phi(p-r)-(p-r)=0 \Longleftrightarrow p-r \in \operatorname{Ker}(\phi-\mathrm{id}) .
\end{gathered}
$$

Analogously, $q-s \in \operatorname{Ker}(\phi-\mathrm{id})$. Since $\operatorname{dim} \operatorname{Ker}(\phi-\mathrm{id})=1$, it follows that $q-s \| p-r$. This is the second statement.

\section{Classification of affine transformations of the plane}

A simple consequence of the fundamental theorem of plane-affine geometry is the following: every affine transformation in the plane is the product of a similarity and an axial affine transformation. Namely, let the affine transformation $F$ be given by

$$
(p, q, r) \mapsto\left(p^{\prime}, q^{\prime}, r^{\prime}\right) .
$$

Let $\chi$ denote a similarity for which $\chi(p)=p^{\prime}$ and $\chi(p)=q^{\prime}$. Then $F$ is the product of $\chi$ and the axial affine transformation $\left[p^{\prime} q^{\prime}, \chi(r) \mapsto r^{\prime}\right]$.

If $F$ has a unique fixed point, then it can be chosen as $p$, thus $p=p^{\prime}$.

THEOREM 3. If affine transformation of the plane has a unique fixed point $p$, then it is the product of a rotation around $p$, a central dilatation with center $p$ and an axial affinity, where $p$ is on the axis. 


$$
\text { "kovacs" — 2006/6/22 — 16:26 — page } 107 \text { — \#7 }
$$

If there are no fixed points, the similarity reduces to a translation, and we have

THEOREM 4. Every affine transformation of the plane without fixed points is the product of a translation and an axial affine transformation.

Proof. As a consequence of the fixed point theorem, in this case

$$
\operatorname{dim} \operatorname{Ker}(\phi-\mathrm{id}) \geq 1
$$

Let $q-p \in \operatorname{Ker}(\phi-\mathrm{id}),\left(p, q \in \mathbb{R}^{2}, p \neq q\right)$. Then $F(q)-F(p)=q-p$, therefore $(p, q)$ can be translated to $(F(p), F(q))$. The translation, determined by $p \mapsto F(p)$, is denoted by $\tau$. Now the required affine transformation can be given as

$$
(p, q, r) \mapsto(F(p), F(q), F(r)) .
$$

This affinity is the product of the translation $\tau$ and the axial affine transformation $[F(p) F(q), \tau(r) \mapsto F(r)]$ (Figure 2.)

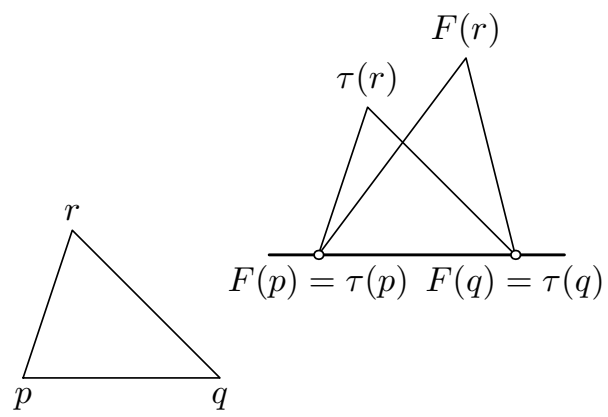

Figure 2. An affine transformation without fixed point is the product of a translation and an axial affinity

\section{The equiaffine case}

An affine transformation of the plane that preserves the area is called equiaffine transformation.

Let $p, q, r$ be non-collinear points in $\mathbb{R}^{2}$. The unique affine transformation that leaves $p$ fixed, while interchanges $q$ and $r$, is called an affine reflection and 


$$
\text { "kovacs" — 2006/6/22 — 16:26 — page } 108 \text { — \#8 }
$$

denoted by $[p, q \leftrightarrow r]$. In addition, let $s$ be the midpoint of the segment $q r$. The above affine reflection leaves fixed every point of $p s$ but no other points. Clearly, every Euclidean reflection is an affine reflection.

Let $p, q, r$ be non-collinear points in $\mathbb{R}^{2}$. The unique affine transformation leaving every point fixed on the line through $p$ and parallel to $q r$ and that takes $q$ to $r$, is denoted by $[p ; q \mapsto r]$ and is called a shear. The shear $[p ; q \mapsto r]$ has the line through $p$ parallel to $q r$ as its set of fixed points.

From the fixed point-construction one can derive a simple proof of Veblen's well-known theorem ([7, Theorem 49 of Chap. III]):

THEOREM 5 (Veblen). Every equiaffine transformation of the plane is the product of two affine reflections.

PROOF. If the equiaffine transformation of the plane differs from a translation, a half-turn or a shear, then it can be given by

$$
(a, b, c, d) \mapsto\left(b, c, c^{\prime}, d^{\prime}\right), \quad a d=d^{\prime} c^{\prime},
$$

where $(a, b, c, d)$ is a parallelogram (see [1, Chap. 13.4]). The fixed point construction is illustrated by Figure 3. Let $n$ be the midpoint of the segment $[a b]$. Join $n$ with the unique fixed point $m$, or in the fixed point free case draw a parallel line through $n$ with $p r$ ( $q s)$. This line is denoted by $t$.

In the case of the unique fixed point, the affinity can be given by

$$
(a, b, m) \mapsto(b, c, m),
$$

moreover in the fixed point free case by

$$
(a, b, d) \mapsto\left(b, c, d^{\prime}\right) .
$$

In both cases the affinity is the composition of the affine reflections $[t, a \leftrightarrow b]$ and $[p, a \leftrightarrow c]$.

The case of a shear can be easily handled: the shear $[p ; q \mapsto r]$ is just the product of the affine reflections $[p ; q \leftrightarrow s]$ and $[p ; s \leftrightarrow r]$, where $s$ is on the image of $q r$ by the half-turn with center $p$.

A translation or a half-turn is the product of two Euclidean reflections, so the statement is obvious.

The equiaffine geometry and the equiaffine group has been extensively studied. A recent approach to the geometric axiomatization of equiaffine geometry is presented in paper [4]. A higher dimensional generalization of Veblen's theorem can be found in [2]. 


$$
\text { "kovacs" — 2006/6/22 — 16:26 — page } 109-\# 9
$$

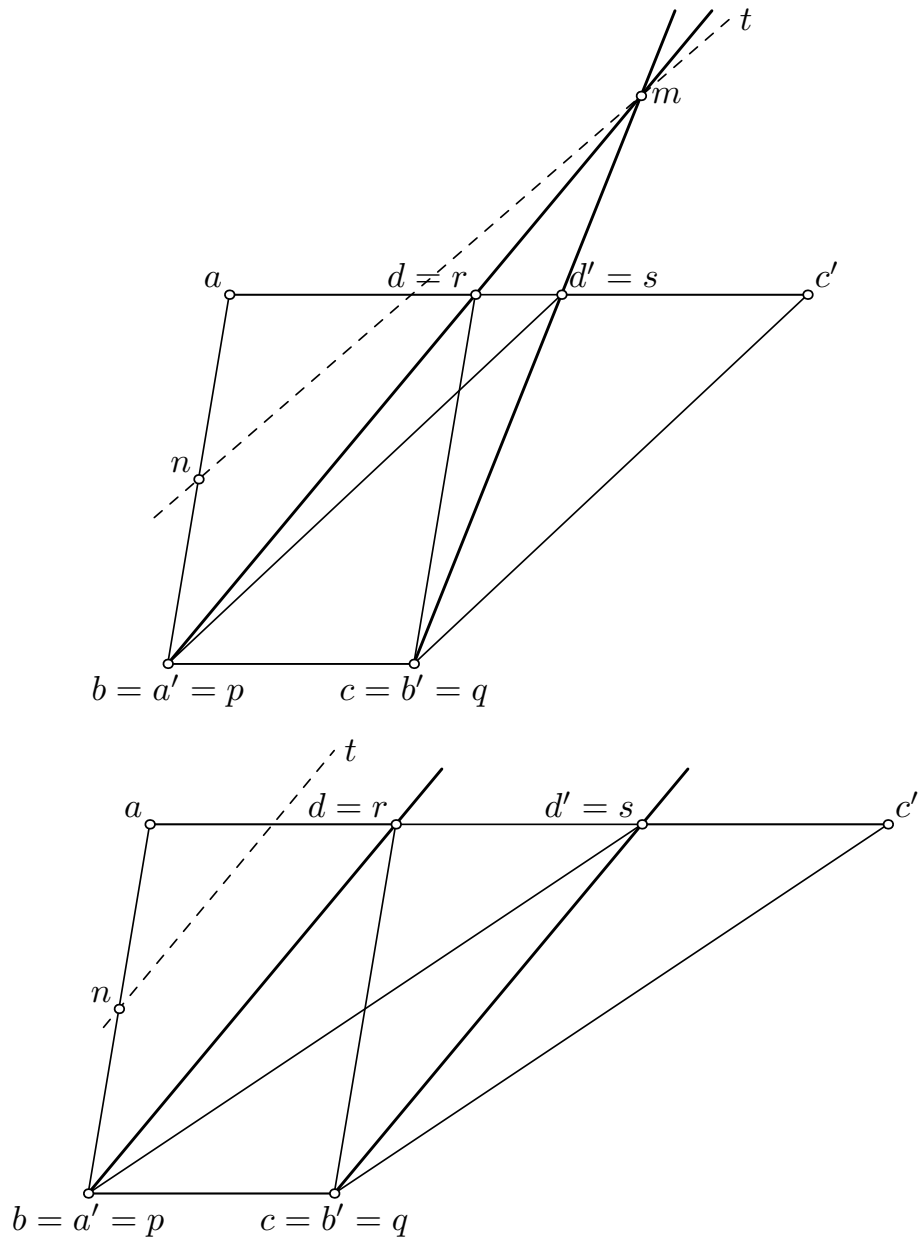

Figure 3

\section{Acknowledgments}

I am deeply indebted to P. T. Nagy (University of Debrecen, Hungary) for inspiring conversations on this topic. I also appreciate the help of J. Horváth (University of Sopron, Hungary) at a very early stage of this paper. His joint work with M. Hollai [3] seems to me the closest in spirit to my own view. 


$$
\text { "kovacs" — 2006/6/22 — 16:26 — page } 110 \text { — \#10 }
$$

\section{References}

[1] H. S. M. Coxeter, Introduction to Geometry, Second edition, John Wiley \& Sons, Inc., 1969.

[2] E. W. Ellers, Decomposition of equiaffinities into reflections, Geometriae Dedicata 6 (1977), 297-304.

[3] M. Hollai and J. Horváth, Affine transformations in the plane and in the space, in: Studies on Mathematical Education, Faculty of Science, ELTE, Hungary, 1979, 21-74 (in Hungarian).

[4] V. Pambuccian, What is plane equiaffine geometry?, Aequationes Mathematicae 66 (2003), 90-99.

[5] P. J. Ryan, Euclidean and non-Euclidean Geometry, Cambridge University Press, 1986.

[6] Z. A. Skopec, On fixpoints of affine mappings, Matematika v skole 3 (1972), 5-10 (in Russian).

[7] O. Veblen and J. W. Young, Projective Geometry, Vol. I., II., Blaisdell Publishing Company, 1965.

ZOLTÁN KOVÁCS

INSTITUTE OF MATHEMATICS AND COMPUTER SCIENCE

COLLEGE OF NYÍREGYHÁZA

H-4401 NYÍREGYHÁZA

P. O. BOX 166

HUNGARY

E-mail: kovacsz@nyf.hu 